\title{
Arbor
}

\section{El Sistema Nacional de Salud y las Políticas de Recursos Humanos: Análisis (critico) y perspectivas de Futuro}

\section{José Luis de Sancho Martín}

Arbor CLXXX, 710 (Febrero 2005), 411-416 pp.

La necesaria brevedad de este artículo me ha hecho situar su inicio en el momento de la plena descentralización de la gestión sanitaria producida en nuestro País al culminarse en Enero de 2002 la transferencia de la gestión sanitaria hasta entonces ejercida para 10 Comunidades Autónomas(desde ahora CC. AA) por el extinto Instituto Nacional de la Salud (desde ahora Insalud), ya olvidado por la mayoría del sector y recordado en justicia por algunos, entre los que me encuentro, por lo que significó en la sanidad española durante más de 20 años.

Se cumplían así las previsiones de la Ley General de Sanidad de 1986 de adoptar un modelo de gestión descentralizada en los territorios autonómicos mediante la génesis de sus propios Servicios de Salud, con competencias de gestión absolutas en el modo de organizar y gestionar los servicios sanitarios, y casi todos los miembros del sector nos felicitamos por ello.

Hecho este breve recuerdo del pasado más reciente, entraré a analizar las perspectivas que sobre la política y la gestión de los recursos humanos (RR. HH desde ahora) ha tenido y tendrá la situación actual del Sistema Nacional de Salud (desde ahora SNS) .

Obviamente, los RR. HH constituyen uno de los pilares fundamentales en los que se han sustentado la eficacia y eficiencia del sistema sanitario de nuestro País, bien situado en el contexto internacional en un honroso séptimo puesto según los indicadores de salud de la población habitualmente utilizados en los países desarrollados. 
La profesionalización de médicos y enfermeras es notable, el sistema de especialización MIR para los facultativos y la formación de la enfermería en las Eścuelas de Enfermería públicas y privadas han sido siempre destacados como unos grandes logros de la sanidad española, y los más recientes programas de formación continuada para estos y otros profesionales están contribuyendo y deben hacerlo más aún en un futuro, a mejorar la cualificación técnica de nuestros profesionales.

Con todo, se plantea ya abiertamente la necesidad de actualizar y revisar el programa MIR para adaptarlo a las nuevas necesidades de especialistas del SNS, con carencias graves en algunas Especialidades tradicionalmente muy demandadas, casos de la Anestesiología y Reanimación o el Radiodiagnóstico, u otras Especialidades emergentes en función de la evolución de los parámetros de supervivencia de los ciudadanos, como la Geriatría, la Oncología, la Genética y alguna otra, o para adecuar la capacitación de los facultativos a las nuevas tecnologías o la gestión sanitaria.

Recientemente se plantea una propuesta de Real Decreto, avalada por la Confederación Estatal de Sindicatos Médicos (CESM), y más concretamente, por la propia Asociación Española de Médicos Internos y Residentes (AEMIR), pretende implantar una nueva regulación para la relación laboral especial de la residencia, donde se dan sensibles avances en determinados aspectos de dicha relación laboral, entre ellos los referentes a los derechos y deberes, como el derecho sindical de participación y negociación de los órganos de representación legal y a determinadas limitaciones actuales respecto de jornada laboral y licencias.

Uno de los primeros problemas planteados en el seno del Consejo Interterritorial del SNS (desde ahora CISNS) nada más transferirse el Insalud, fue el conflicto sobre la titularidad de la acreditación de la formación continuada y la competencia del desarrollo posterior de acciones formativas.

Las CC. AA reclamaban para si tal titularidad a través del previo acuerdo de la Comisión de RR. HH del CISNS, que sin embargo no fue respetado por el propio Ministerio de Sanidad y Consumo (desde ahora MSC), al firmarse un convenio con cinco Colegios profesionales que también se arrogaban dicha titularidad.

Este hecho, que pude vivir en primera instancia, significó de facto el primer enfrentamiento político entre los Consejeros de Salud socialistas y conservadores, abandonando aquellos el Plenario de dicho CISNS hasta que no se devolviera a la Comisión de RR. HH el protagonismo acreditador de la formación continuada y a las CC. AA el protagonismo ejecutor de estas políticas. 
Ciertamente, con posterioridad se han producido ausencias y abandonos de otros Plenarios del CISNS, ya con Gobierno socialista por parte de los Consejeros de Salud conservadores, lo que desde una visión crítica no favorece en absoluto un desarrollo armónico de los grandes retos pendientes de este nuevo escenario del SNS surgido en 2002: dotarse de un observatorio único y un sistema de información común para todo el SNS, integrador de conocimiento de lo que pasa realmente en la asistencia sanitaria del país, desarrollar políticas de prestaciones consensuadas articulando adecuadamente su incorporación y financiación por el Sistema, unificar criterios e indicadores básicos de eficiencia y calidad que posibiliten la formulación de un auténtico «benchmark» de servicios sanitarios hecho desde el propio Sistema, respondiendo efectivamente a las necesidades de los servicios sanitarios y las demandas de los ciudadanos o para articular políticas de atención socio-sanitaria igualmente efectivas.

Pero retornando a las políticas de RR. HH, si bien es necesario mencionar como un acierto la aprobación de un nuevo Estatuto Marco de las profesiones sanitarias, sustituto de aquellos Estatutos de los años 60, preconstitucionales y desfasados, predomina el desconcierto. Citaré algunos ejemplos., que me gusta calificar de «nuevas oportunidades perdidas» por el SNS.

¿Cómo es posible que todavía se esté hablando de articular una única carrera profesional cuando ya 5 CC.AA (Navarra, Cataluña, Canarias, Andalucía y País Vasco) tienen implantada o en fase avanzada de implantación la que fuera demandada durante años, diseñada y tutelada por el MSC.? Madrid, Galicia, Valencia y Extremadura lo están discutiendo con los respectivos interlocutores y el resto como Murcia, CastillaLeón además de Ceuta y Melilla se los están planteando.

Armonizar desde el MSC los posibles 17 modelos de carrrera profesional es para mi una incógnita de difícil solución. El hecho cierto es que si un facultativo o enfermera trabaja en según qué Comunidad Autónoma goza ya de un reconocimiento profesional y salarial que hoy no tienen sus colegas de igual titulación y resposabilidad en otras CC.AA.

En cualquiera de las posibles carreras profesionales creo que se está difuminando la posibilidad real de discriminar positivamente a los profesionales más relevantes, eficientes e implicados con el sector sanitario público que podría haber tenido este nuevo instrumento, convirtiéndose de facto en un incremento salarial adicional al incremento anual basado en el IPC, que siempre ha sido insatisfactorio para los profesionales. 
Algo similar sucede con la política seguida por algunas CC. AA, hasta la fecha Cataluña, Andalucía y Valencia, de aplicar el nuevo Estatuto Marco del personal estatutario del SNS de diciembre de 2003, consecuencia de la Ley de Cohesión y Calidad de mayo de 2003, en su apartado de jubilaciones de manera forzosa a los 65 años, mientras otras CC. $\mathrm{AA}$, lo harán opcionalmente por los propios interesados a los 65 o los 70 años, casos de Madrid, Galicia, y las dos Castillas, en este último caso con partidos gobernantes de signo distinto, y aún quedan otras CC. AA en la incertidumbre.

Puede ocurrir que un facultativo que haya concurrido en el concurso de traslados de la Oferta Pública de Empleo (OPE) del 2001 del Insalud desde Andalucía a Madrid incremente su vida laboral en 5 años, y viceversa, si se le hubiera ocurrido hacerlo desde Madrid a Valencia, ambas gobernadas por cierto por un mismo partido político, reduciría su expectativa laboral.

Aún así, considero acertadas las políticas llevadas a cabo en este sentido por Cataluña y Andalucía, por cuanto significan una oportunidad de recambio generacional en los centros sanitarios, particularmente en los Hospitales, donde la plaza en propiedad y la vitalicidad de las jefaturas particularmente, han significado en muchos casos un lastre insalvable para la mejora de los Servicios asistenciales y un freno a la innovación o la gestión clínica como nuevo instrumento de cambio organizativo.

Bien es cierto que se han desaprovechado en el pasado por los propios Servicios de salud normativas de carácter estatal que modificaban estos aspectos, que permitían la evaluación periódica de la responsabilidad de las jefaturas de servicio, y tampoco se hizo en algunos casos, siendo el mismo Insalud un ejemplo de ello. En suma, otra "oportunidad perdida».

He mencionado antes un hecho que creo será algún día reconocido por el sector sanitario de este país, como uno de los logros de la etapa final del Insalud, la OPE del año 2001, después de más de 10 años sin convocatorias de estabilización del empleo sanitario y no sanitario, aún en fase de resolución después de más de 3 años desde su convocatoria en el Boletín Oficial del Estado. Tal vez otros responsables sanitarios no la hubieran convocado nunca ante la proximidad de la transferencia de dicho organismo, dejándolo a criterio de cada Servicio de salud o hubiesen optado por la laboralización a partir de una fecha cierta, reservando los derechos adquiridos de los estatutarios con plaza en propiedad hasta su jubilación, y esta última opción fue valorada intensamente, pero presiones políticas y sindicales y otras causas impidieron concretarla.

Otro aspecto no adecuadamente resuelto en el sector ha sido la introducción de las políticas de incentivación ligadas a instrumentos de ges- 


\section{El Sistema Nacional de Salud y las Políticas de Recursos...}

tión y a la mejora de la productividad de los servicios, como ocurre en otros sectores empresariales y productivos, a través de la denominada «Dirección por objetivos»(DPO) o el concepto más comúnmente conocido por "productividad variable».

Aunque han existido y existen iniciativas en este sentido en varios Servicios de salud, existe el riesgo de que puedan acabar convirtiéndose en incrementos salariales más o menos estables, no discriminando adecuadamente a los más eficientes, si bien son interesantes y esperanzadoras las más recientes experiencias de modelos de gestión alternativos al del hospital tradicional, ya sean Fundaciones sanitarias, ya probadas en el territorio Insalud, Empresas públicas, igualmente probadas en Andalucía y País Vasco, o Consorcios, casi específicos de Cataluña, que ya desde su creación han contemplado fórmulas de contratación de los profesionales con componentes retributivos fijo( $70-75 \%)$ y variable (30-25 \%), vinculado este último al logro de los objetivos anuales pactados, y también las recientes experiencias de Institutos o Unidades de gestión clínica, que han establecido sistemas de incentivación económica o de otra índole en el acuerdo o contrato de gestión anual.

Dado que no cabe esperar a corto plazo grandes incrementos retributivos para los profesionales del sector sanitario, reorientar en todo caso las políticas retributivas de los profesionales del SNS, orientándolas más a la compensación del desempeño y la competencia profesional, incrementando el componente variable en detrimento del fijo, será en mi opinión uno de los grandes retos de los Servicios de salud, al que seguramente se opondrán los sindicatos del sector, defensores de la sanidad pública todos ellos, pero poco sensibles a aceptar este tipo de planteamientos.

Otro aspecto a considerar es la creciente aplicación de políticas de prevención de riesgos laborales derivadas de la Ley del mismo enunciado del año 1995, lo que debe comportar un esfuerzo importante en su desarrollo por la organizaciones sanitarias, a la vez que representan una oportunidad de mejora de las condiciones laborales de los profesionales y de sus competencias lo que incidirá positivamente en la calidad de la prestación asistencial.

La gestión de RR. HH por los Servicios de salud y centros sanitarios debe encontrar fórmulas de flexibilizar sus sistemas de contratación de profesionales, particularmente los de mayor cualificación, médicos y enfermeras, aunque no exclusivamente, permitiendo la incorporación a sus plantillas de los mejores, sea cual sea su procedencia e individualizando con "contratos a medida»de las necesidades de cada centro la incorporación de aquellos. Este aspecto clave es hoy una barrera casi insalvable 
que dificulta el liderazgo de algunos programas y proyectos relevantes, especialmente en el campo de la investigación pero también para un mayor y más rápido desarrollo de la gestión clínica en nuestro país, por cierto y siempre desde mi punto de vista, otra posible «oportunidad perdida,», especialmente para los hospitales de nivel referencial, de no corregirse pronto estos obstáculos.

Otro aspecto que me parece relevante es la necesidad de una mayor implicación de los profesionales en la gestión de los centros y servicios sanitarios, que debería acompañarse de un imprescindible acercamiento de posiciones entre gestores "profesionales» y gestores "clínicos», desde posiciones de confianza mutua hoy casi inexistente. Ciertamente la "politización» excesiva de los directivos de primer nivel aún existente en nuestro país ayuda poco a alcanzar este logro, pero ha de trabajarse en esta línea de cambio en el futuro inmediato. Profesionalizar más a unos y otros es otro reto pendiente del SNS, si bien proliferan las acciones de carácter formativo en gestión y es cada día mayor la concienciación de los profesionales sanitarios de la necesidad de incorporar cierto bagaje gestor a sus conocimientos.

El reciente nuevo marco legislativo del que se ha dotado la sanidad española, Ley 16 /2003 de Cohesión y calidad del SNS, la Ley 44/2003 de Ordenación de las profesiones sanitarias, y la Ley 55/2003 del Estatuto Marco del personal estatutario de los Servicios de salud en sus ulteriores desarrollos, debería posibilitar sensibles avances en algunos de los aspectos comentados en este artículo y otros ,que en el contexto de las políticas de recursos humanos, pudieran abordarse y que por la necesaria brevedad de este artículo no he abordado. 